\title{
Atypical skull base osteomyelitis in the intensive care unit: A case report
}

\author{
Pradeep Hiremath ${ }^{1}$, Pradeep Rangappa*1, Ipe Jacob ${ }^{1}$, Sriram Patwari ${ }^{2}$, Karthik Rao ${ }^{1}$ \\ ${ }^{1}$ Department of Intensive Care, Columbia Asia Referral Hospital Yeshwanthpur, Bangalore, India \\ ${ }^{2}$ Department of Radiology, Columbia Asia Referral Hospital Yeshwanthpur, Bangalore, India
}

Received: October 10, 2017

Accepted: December 5, 2017

Online Published: December 17, 2017

DOI: $10.5430 /$ ijdi.v5n1p15

URL: https://doi.org/10.5430/ijdi.v5n1p15

\begin{abstract}
Base of skull osteomyelitis is commonly seen as a complication of malignant otitis externa, involving the temporal bone. It initially presents with aural symptoms such as ear ache and discharge and cranial nerve palsies. We report an atypical presentation of skull base osteomyelitis that did not show signs of otitis externa. The patient presented with severe headache, drowsiness and signs of bulbar weakness including pooling of oropharyngeal secretions. Computerized Tomography (CT) and Magnetic Resonance Imaging (MRI) studies showed a bony erosion of the left side of base of skull involving the sphenoid bone and surrounding foramina, left sided coalescent mastoiditis and inflammation of the left parapharyngeal space. There was also inflammation of the tissues encasing the internal carotid artery and jugular veins and thrombosis of left jugular vein. These imaging findings along with cranial nerve palsies were suggestive of malignancy. However, tissue biopsy was negative for malignancy. The growth of Pseudomonas aeruginosa in the biopsy material as well as nasopharyngeal and blood cultures along with elevated Erythrocyte Sedimentation Rate (ESR) and C-Reactive Protein (CRP) levels led to a diagnosis of base of skull osteomyelitis. Hence diagnosis in such cases requires that biopsy material be sent for microbiological analysis, in addition to histology.
\end{abstract}

Key Words: Malignant otitis externa, Bulbar weakness, Malignancy, Tissue biopsy, Pseudomonas aeruginosa

\section{INTRODUCTION}

Base of skull osteomyelitis is an uncommon, life threatening disorder.It is usually seen in malignant otitis externa (MOE), an infection affecting the external auditory canal and temporal bone. It initially involves the lateral part of the temporal bone and extends to the centre of base of skull, specifically to the petrous apex and the clivus. ${ }^{[1]}$

As such, the patient initially presents with auditory symptoms including persistent ear discharge, ear pain and loss of hearing, or with cranial nerve palsies involving the oculomotor (III), trigeminal (V), abducens (VI), facial (VII), vestibulocochlear (VIII), glossopharyngeal (IX), vagus (X), accessory (XI) and hypoglossal (XII). ${ }^{[2,3]}$ This disease usually occurs in diabetes mellitus and elderly patients with high cerebrocardiovascular risks.

The challenge posed to the clinician lies in the similar physical and imaging findings in base of skull osteomyelitis and malignancy. Involvement of both cranial nerve VI and the lower cranial nerves (IX, X, XI) may suggest a clival pathology. ${ }^{[4]}$ Ophthalmoplegia, due to sixth nerve paresis and paresis of cranial nerve XII are suggestive of malignancy, most commonly of the nasopharynx. ${ }^{[5]}$ India.

Published by Sciedu Press 
Imaging findings may frequently mimic malignancy, especially erosion of the basilar process of occipital or apical portion of petrous bone, along with swelling of soft tissues at the base of skull. Tissue biopsy samples are usually sent for histology only and are often non-conclusive. As this does not correlate with the gross changes seen on imaging, an initial negative biopsy is concluded to be a sampling error, leading to a further delay in diagnosis. Hence, microbiological analysis of biopsy material, including fungal and mycobacterial cultures, in addition to histology, may lead to a correct diagnosis and earlier institution of antibiotics. Currently, biopsy for microbiological culture is the ideal method of diagnosis in cases of suspected skull base osteomyelitis. Once diagnosed and treated with appropriate antibiotics, it carries a good prognosis.

\section{CASE REPORT}

A 58 years old male with diabetes mellitus, hypertension and chronic kidney disease was admitted with a history of headache, hoarseness of voice, dysphagia, pooling of oropharyngeal secretions, excessive cough and tiredness. On arrival, he was unresponsive, hypotensive and gasping. He was intubated and mechanically ventilated and started on antibiotics for possible aspiration pneumonia. His sensorium improved and he was extubated after three days. However, he had persistent dysphagia and poor cough reflex, needing frequent oral suctioning. Two days later, his sensorium worsened and hence he was re-intubated. He underwent tracheostomy for airway protection in view of the bulbar weakness.

CT and MRI scans of brain showed left parapharyngeal space inflammation and coalescent mastoiditis. Antero-medially, the lesion extended to the left lateral parapharyngeal wall. Posteriorly, the lesion invaded the body and greater wing of the sphenoid, clivus and left petrous apex with a permeative pattern of bone destruction (see Figure 1). The foramina ovale and spinosum on the left side were widened (see Figure 2). Along with the bony destruction, there was an enhancing ill-defined soft tissue on the left side of skull base, encasing the internal carotid artery and jugular veins (see Figure 3). The soft tissue was also seen to extend to the left hypoglossal canal and jugular fossa.

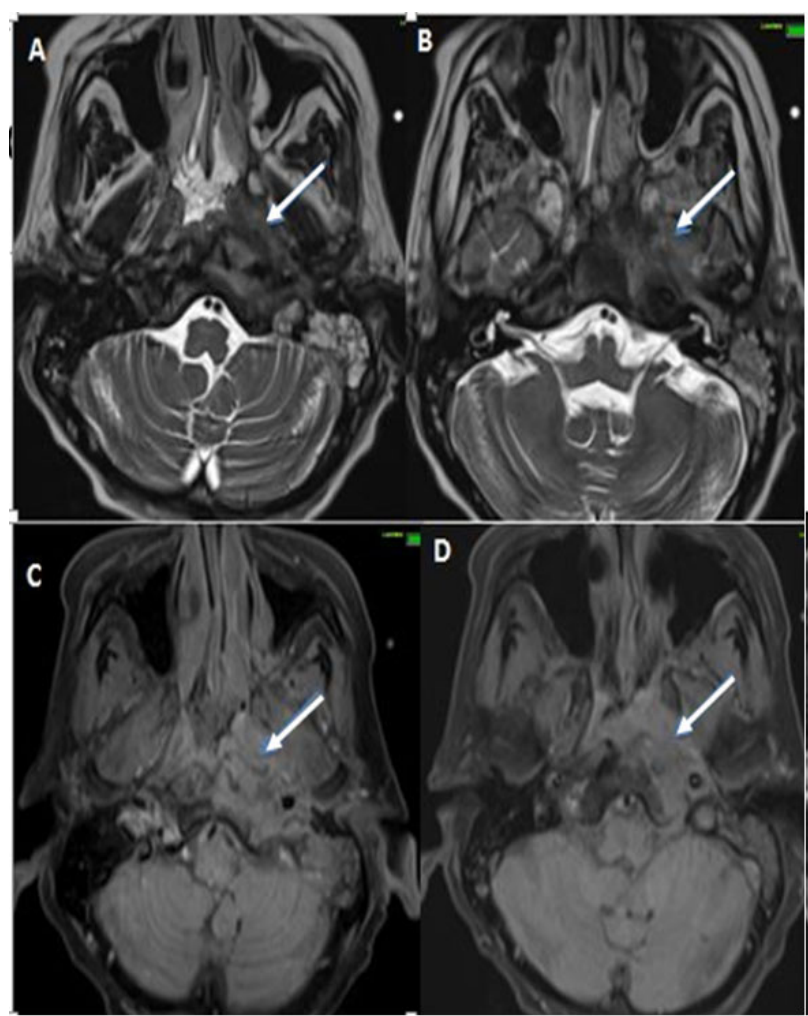

Fig 1A

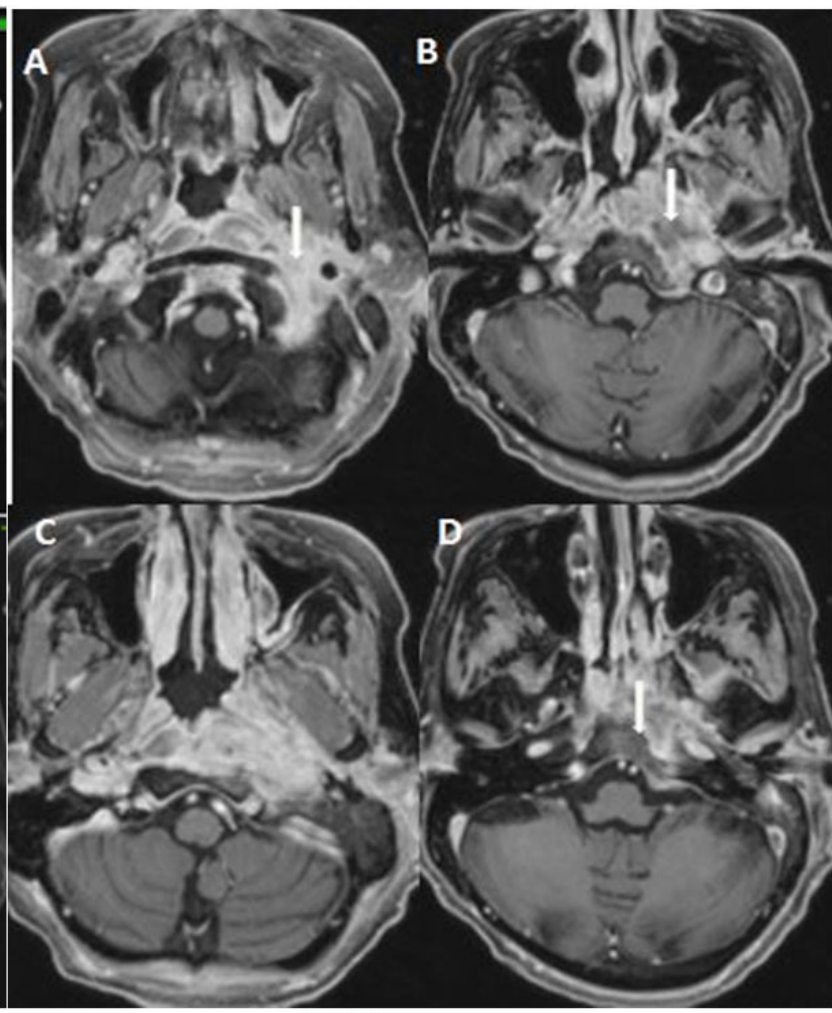

Fig 1B

Figure 1. Fig 1A: MRI Precontrast Axial T2 (A \& B) and axial fat sat $T 1(C \& D)$ images demonstrate ill-defined $T 2$ hypointense and $\mathrm{T} 1$ isointense lesion (arrow) seen in the left side of base of skull. There is fluid filled opacification of left mastoid air cells. Fig 1B: MRI Postcontrast Post contrast axial T1 images (A to D) demonstrates enhancement in left side of the skull base with minimal collection (image B arrow). There is involvement of hypoglossal and jugular canals with abnormal enhancement seen in left side of clivus (image D). 


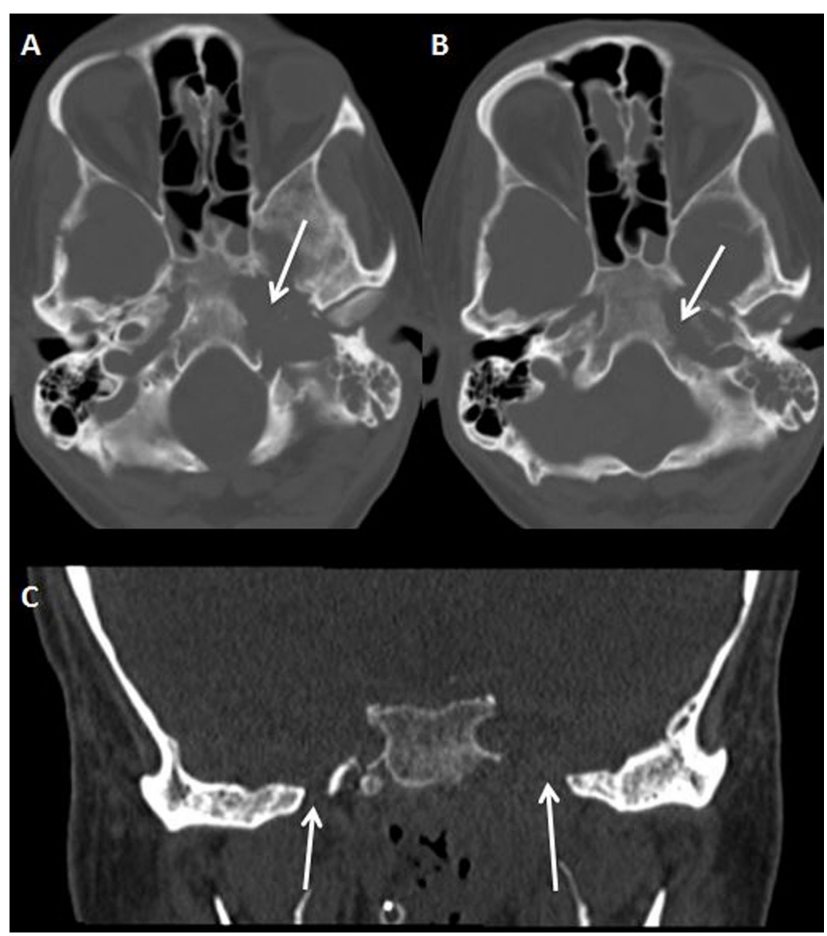

Figure 2. CT BONE WINDOW: Axial CT images (A, B) demonstrate erosion of left side of skull base involving left side of clivus. Coronal CT image $(\mathrm{C})$ demonstrates widening

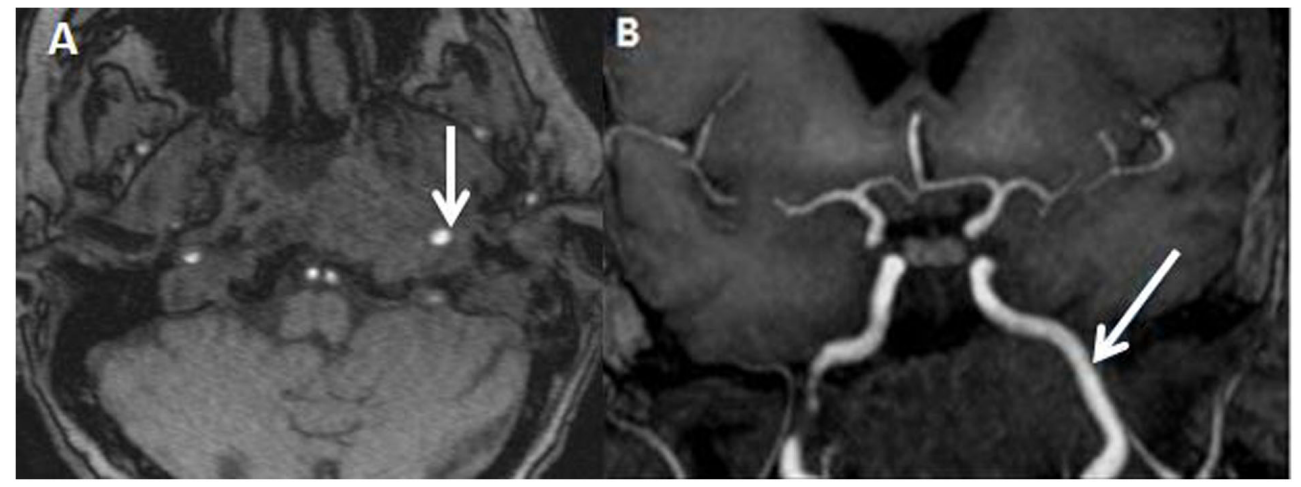

Figure 3. Axial source images of Time of Flight cerebral angiogram (A) and Coronal MIP image (B) demonstrate encasement of left cervical internal carotid artery without any narrowing

As part of the work up to rule out malignancy as well as for therapeutic reasons, he underwent functional endoscopic sinus surgery with parapharyngeal space drainage. It also showed a mass behind the left fossa of Rosenmuller, from which a biopsy was taken and sent for histopathology and culture. The cultures from the parapharyngeal abscess and tissue biopsy grew multi drug resistant Pseudomonas aeruginosa, sensitive only to Colistin and Amikacin and antibiotics were changed accordingly. Histopathology was negative for malignancy. Antibiotics were given for a total of 12 weeks. He showed a gradual but consistent neurological improvement over this period. His swallowing and cough reflexes recovered and his tracheostomy was closed. A MRI scan Published by Sciedu Press showed resolution of the osteomyelitis with a striking disappearance of the skull base lesion.

\section{Discussion}

Base of skull osteomyelitis is a rare and potentially fatal condition if not recognized early and properly treated. ${ }^{[1]}$ It often presents with non-specific symptoms such as persistent headache, with the eventual development of cranial neuropathy. It is most commonly seen in middle-aged or elderly males, with an underlying immunocompromised state such as diabetes mellitus, corticosteroid use, Human Immunodeficiency Virus (HIV) infection or chronic inflammatory sphenoid sinus disease. 
In this case, the patient presented with bulbar symptoms, including dysphagia and pooling of oropharyngeal secretions, but did not have any aural symptoms. The erosion seen on the base of skull did not involve the external auditory canal. Typically, skull base osteomyelitis is preceded by a destructive malignant otitis externa, but atypical cases which present without aural symptoms are also reported. ${ }^{[1,2,6]}$ In a study of forty-two such atypical cases, headaches, facial pain and palsy of cranial nerves were the commonest presenting features. ${ }^{[3]}$

The radiological findings of bony erosion of base of skull and soft tissue swellings often raise a suspicion of a malignant process such as nasopharyngeal carcinoma or skull base metastases. However, the latter would generally result in a more focussed area of bony destruction, rather than the diffuse pattern seen in the present case. Lymphoma, however, may present with similar imaging findings as in skull base osteomyelitis. Leukemia, on the other hand, may present with an even more widespread pattern involving the clivus, calvarium and cervical spine, but would be unlikely to show similar soft tissue changes. Nasopharyngeal carcinoma usually has a distinct mucosal mass lesion, which was absent in the present case.

It is not possible to differentiate malignancy from an infective process through imaging alone and hence it is necessary to perform a biopsy for microbiological analysis. Another useful discriminator is the acute-phase reactants ESR and CRP, which are elevated in inflammatory conditions and not in malignancy. Monitoring ESR and CRP can also guide the duration of antibiotic therapy. Its normalization would be a good indicator of resolution of the infection.

Base of skull osteomyelitis carries a mortality rate of $10 \%$, with one-third of patients showing long-term neurologic deficits, despite appropriate management. ${ }^{[3]}$ The commonest causative organism is Pseudomonas aeruginosa and less commonly, fungal and mixed bacterial infections. ${ }^{[2,3]}$

Complications of skull base osteomyelitis include thrombosis of the cerebral venous sinuses and the internal jugular vein. There is a potential risk of carotid artery thrombosis as well. ${ }^{[7]}$ In our case, there was partial thrombosis of the left jugular vein, left sigmoid sinus and lateral third of the transverse sinus (see Figure 4). Infection and inflammation associated with osteomyelitis may lead to an inflammatory arteritis, with formation of plaques prone to rupture. ${ }^{[7]}$

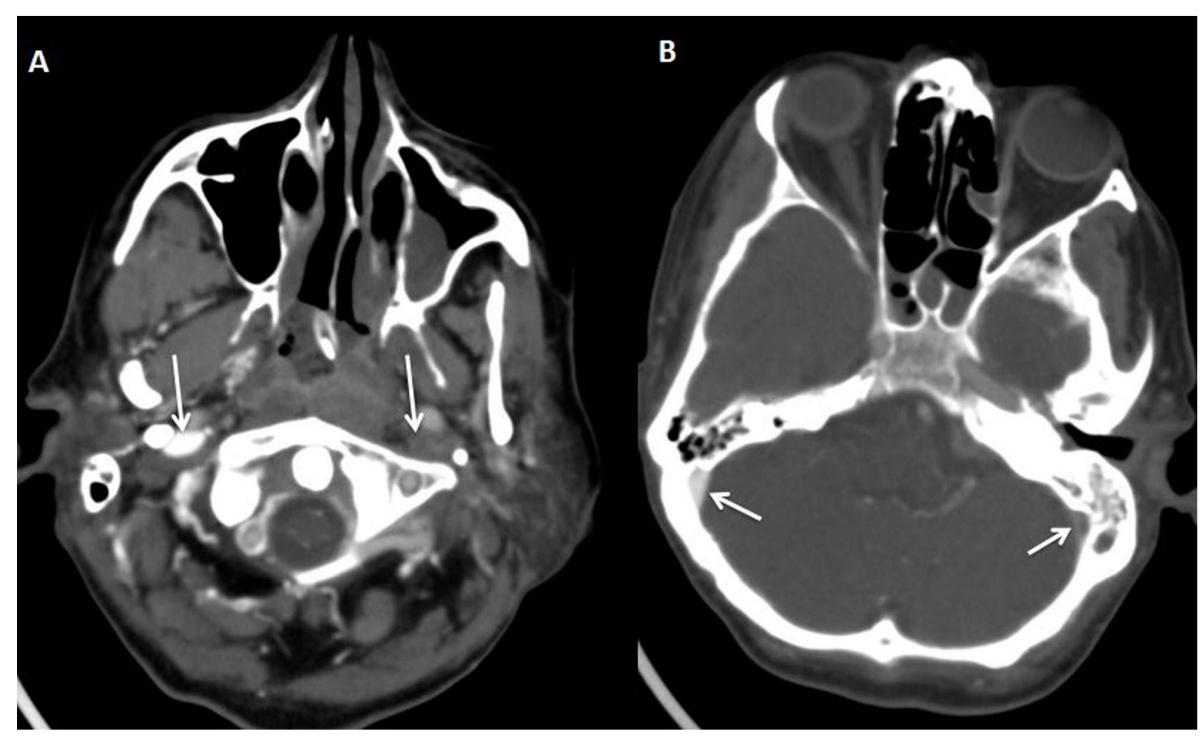

Figure 4. SOFT TISSUE WINDOW: Axial Post contrast CT images (A, B) demonstrate thrombosis of left internal jugular vein and sigmoid sinus. Normal contrast filling right internal jugular vein and sigmoid sinus seen on right

The total duration of antibiotic therapy for skull base osteomyelitis remains undefined at present, although 6 weeks of intravenous antibiotics for the treatment of native vertebral osteomyelitis, as suggested by the Infectious Diseases Society of America, may be taken as a guideline. ${ }^{[8]}$ The Bone Infection Unit in Oxford, United Kingdom, recommends both intravenous antibiotics up to 6 weeks followed by oral medications for 6 to 12 months, depending on clinical improvement. ${ }^{[9]}$ Hyperbaric oxygen therapy as an adjuvant may be beneficial. The latter has been shown to improve outcome in cases of malignant otitis externa by reversing tissue hypoxia, promoting the phagocytosis of aerobic microorganisms and neomicroangiogenesis. ${ }^{[10]}$ Hyperbaric oxygen therapy, however, is limited in its availability. 
In the case presented here, intravenous antibiotics were given for 14 days but did not lead to clinical improvement. Hence, a further course of intravenous antibiotics for 12 weeks was initiated. The antibiotics were discontinued after remission of cranial nerve complications and normalisation of ESR and CRP levels. A repeat MRI scan showed resolution of the earlier lesions. In contrast, other studies have shown that the lesions seen on MRI may persist even after remission of symptoms. ${ }^{[11]}$ Hence, MRI serves in diagnosis but may not determine the duration of therapy. MRI with gadolinium contrast may be effective in evaluating the exact area of skull base osteomyelitis, although it could not be used in the present case due to renal failure. ${ }^{[7]}$
In conclusion, in this case of osteomyelitis of base of skull, the inflammation also involved multiple cranial nerves resulting in bulbar weakness. Imaging findings may represent a red herring and require to be substantiated by a prompt tissue biopsy and culture to arrive at a diagnosis. We suggest that the finding of cranial nerve palsies in the immunocompromised or elderly, coupled with radiological findings of bony erosion of central skull base, be concurrently investigated for skull base osteomyelitis as well as malignancy.

\section{CONFlicts OF InTEREST Disclosure}

The authors have no conflict of interest related to this publication.

\section{REFERENCES}

[1] Chandler JR, Grobman L, Quencer R, et al. Osteomyelitis of the base of the skull. Laryngoscope. 1986; 96(3): 245-251. PMid:3485233 https://doi.org/10.1288/00005537-198603000-00003

[2] Ridder GJ, Breunig C, Kaminsky J, et al. Pfeiffer. Central skull base osteomyelitis: new insights and implications for diagnosis and treatment. European Archives of Oto-Rhino-Laryngology. 2015; 272(5): 1269-1276. PMid:25381580 https://doi.org/10.100 7/s00405-014-3390-y

[3] Johnson AK, Batra PS. Central skull base osteomyelitis: an emerging clinical entity. The Laryngoscope. 2014; 124(5): 1083-1087. PMid:24115113 https://doi.org/10.1002/lary. 24440

[4] Kulkarni S, Lee A, Lee JH. Sixth and tenth nerve palsy secondary to pseudomonas infection of the skull base. Am J Ophthalmol. 2005; 139(5): 918-920. PMid:15860304 https://doi.org/10.1016/j. ajo. 2004.09.021

[5] Keane JR. Combined VIth and XIIth cranial nerve palsies: A clival syndrome. Neurology. 2000; 54: 1540-1541. PMid:10751279 https://doi.org/10.1212/WNL.54.7.1540

[6] Clark MPA, Pretorius PM, Byren I, et al. Central or atypical skull base osteomyelitis: diagnosis and treatment. Skull Base. 2009; 19(4): 247254. PMid:20046592 https://doi.org/10.1055/s-0028-111 5325
[7] Miyabe H, Uno A, Nakajima T, et al. A Case of Skull Base Osteomyelitis with Multiple Cerebral Infarction. Case Reports in Otolaryngology. 2016. PMid:27597916 https : //doi.org/10.1155/ 2016/9252361

[8] Berbari EF, Kanj SS, Kowalski TJ, et al. 2015 Infectious Diseases Society of America (IDSA) Clinical practice guidelines for the diagnosis and treatment of native vertebral osteomyelitis in adults. Clinical Infectious Diseases. 2015; 61(6): e26-e46. PMid:26229122 https://doi.org/10.1093/cid/civ482

[9] Skull B. Central or atypical skull base osteomyelitis: diagnosis and treatment. Clark MP1, Pretorius PM, Byren I, Milford CA. 2009 Jul; 19(4): 247-54. https://doi.org/10.1055/s-0028-1115325

[10] Davis JC, Gates GA, Lerner C, et al. Adjuvant hyperbaric oxygen in malignant external otitis. Arch Otolaryngol Head Neck Surg. 1992; 118: 89-93. PMid:1728284 https://doi.org/10.1001/archot ol.1992.01880010093022

[11] Adams A, Offiah C. Central skull base osteomyelitis as a complication of necrotizing otitis externa: imaging findings, complications, and challenges of diagnosis. Clinical Radiology. 2012; 67(10): e7-e16. PMid:22483946 https ://doi.org/10.1016/j.crad.2 012.02 .004 